\title{
Novel Methodology to Evaluate Renal Cysts in Polycystic Kidney Disease
}

\author{
Kyongtae T. Bae ${ }^{a}$ Hongliang Sun ${ }^{a}$ June Goo Lee ${ }^{a}$ Kyungsoo Bae ${ }^{i}$ \\ Jinhong Wang ${ }^{\text {a }}$ Cheng Tao ${ }^{\text {a }}$ Arlene B. Chapman ${ }^{c}$ Vicente E. Torres ${ }^{d}$ \\ Jared J. Grantham ${ }^{\text {e }}$ Michal Mrug $^{f}$ William M. Bennett ${ }^{g}$ Michael F. Flessner ${ }^{h}$ \\ Doug P. Landsittel ${ }^{\text {b }}$ for the Consortium for Radiologic Imaging Studies of \\ Polycystic Kidney Disease
}

\begin{abstract}
Departments of a Radiology and ${ }^{\mathrm{b}}$ Internal Medicine, University of Pittsburgh School of Medicine, Pittsburgh, Pa., 'Department of Internal Medicine, Emory University School of Medicine, Atlanta, Ga., 'Department of Internal Medicine, Mayo College of Medicine, Rochester, Minn., ' Department of Internal Medicine, Kansas University Medical Center, Kansas City, Kans., fDivision of Nephrology, University of Alabama, Birmingham, Ala., ' Legacy Good Samaritan Hospital, Portland, Oreg., h National Institute of Diabetes and Digestive and Kidney Diseases, National Institutes of Health, Bethesda, Md., USA; 'Department of Radiology, Gyeongsang National University School of Medicine, Jinju, South Korea
\end{abstract}

\section{Key Words}

Kidney · Polycystic kidney disease · Renal cysts ·

Magnetic resonance imaging $\cdot$ Segmentation

\begin{abstract}
Aim: To develop and assess a semiautomated method for segmenting and counting individual renal cysts from midslice MR images in patients with autosomal dominant polycystic kidney disease (ADPKD). Methods: A semiautomated method was developed to segment and count individual renal cysts from mid-slice MR images in 241 subjects with ADPKD from the Consortium for Radiologic Imaging Studies of Polycystic Kidney Disease. For each subject, a mid-slice MR image was selected from each set of coronal T2-weighted MR images covering the entire kidney. The selected midslice image was processed with the semiautomated method to segment and count individual renal cysts. The number of cysts from the mid-slice image of each kidney was also mea-
\end{abstract}

sured by manual counting. The level of agreement between the semiautomated and manual cyst counts was compared using intraclass correlation (ICC) and a Bland-Altman plot. Results: Individual renal cysts were successfully segmented using the semiautomated method in all 241 cases. The number of cysts in each kidney measured with the semiautomated and manual counting methods correlated well (ICC $=0.96$ for the right or left kidney), with a small average difference $(-0.52$, with higher semiautomated counts, for the right kidney, and 0.13 , with higher manual counts, for the left kidney) in the semiautomated method. However, there was substantial variation in a small number of subjects; 6 of 241 participants $(2.5 \%)$ had a difference in the total cyst count of more than 15. Conclusion: We have developed a semiautomated method to segment individual renal cysts from mid-slice MR images in ADPKD kidneys as a quantitative indicator of characterization and disease progression of ADPKD.

(c) 2014 S. Karger AG, Basel

\section{KARGER}

E-Mail karger@karger.com

www.karger.com/ajn (c) 2014 S. Karger AG, Basel

0250-8095/14/0393-0210\$39.50/0
K.T. Bae, MD, PhD

Department of Radiology, University of Pittsburgh School of Medicine Presbyterian South Tower, Room 3950 200 Lothrop St, Pittsburgh, PA 15213 (USA)

E-Mailbaek@upmc.edu 


\section{Introduction}

Autosomal dominant polycystic kidney disease (ADPKD), the most common renal genetic disorder, is characterized by the progressive development and expansion of renal cysts. The decline in renal function in ADPKD correlates strongly with the severity and growth of these renal cysts [1]. In severe cases, cysts replace most of the functional parenchyma, leading to end-stage renal disease [2].

To study the relationship between kidney morphology and function in a prospective, longitudinal ADPKD cohort, we established the Consortium for Radiologic Imaging Studies of Polycystic Kidney Disease (CRISP) [3]. The data collected in this study, including MR imaging, renal function and biomarkers relevant to the early course of ADPKD, showed that MR imaging measurement of kidney volume is more sensitive than glomerular filtration rate measurement in evaluating the yearly progression of ADPKD and that the growth seen in the kidney volume stems directly from increases in renal cyst volume [4].

In addition to total kidney and cyst volumes, the number of individual cysts in each kidney also provides important information about the characteristics and progression of ADPKD. For example, differences in kidney morphology between PKD1 and PKD2 genotypes are likely due to the earlier development rather than faster volumetric growth of cysts in PKD1 kidneys [5]. Generally, the number of individual cysts in each kidney is determined by manually counting them on the mid-section of an MR image set. Although this approach is straightforward, manual counting is time-consuming and labor-intensive, particularly in large kidneys with numerous cysts. Furthermore, it is extremely laborious to segment (i.e. to identify and partition an image into semantically interpretable regions) each cyst from the background renal parenchyma by manual delineation of individual cysts. We recently published a 3-dimensional (3D) semiautomated method to segment renal cysts from entire volumetric MR slices [6]. However, this approach was exceedingly complex and successful only for ADPKD kidneys with mild and moderate cystic burdens. Thus, to overcome the limitations of manual and 3D segmentation of renal cysts, in the current study we developed and evaluated a semiautomated method for segmenting and counting individual renal cysts from mid-slice (2-dimensional, 2D) MR images in patients with ADPKD.

Renal Cyst Evaluation in ADPKD

\section{Materials and Methods}

The study protocol for the CRISP study (clinical trials registration: NCT01039987, registration date: December 23, 2009) has been previously described $[3,4,7]$ and was approved by the institutional review board at each participating clinical center. Informed consent was obtained from all subjects who participated in the CRISP study.

\section{Participants and MR Imaging}

In the CRISP study launched in 1999, 241 ADPKD subjects between 15 and 46 years old with relatively intact renal function were recruited. The clinical characteristics of the cohort and a detailed study protocol have been published previously $[3,4,7]$. MR images of kidneys were obtained at a fixed slice thickness of $3 \mathrm{~mm}$ in the coronal plane. Both 3D spoiled gradient interpolated T1weighted images without fat saturation and single-shot fast spin echo T2-weighted images with fat saturation were acquired [3].

\section{Manual Middle-Section Cyst Counting}

The T2-weighted MR images of the abdomen from each of the 241 patients were reviewed and cropped to generate separate images of the right and left hemiabdomen. From the cropped image set of the hemiabdomen, the mid-slice of the kidney was selected by reviewing and scrolling the image set. The kidney boundary was detected and segmented using image editing software (Analyze, version 10.0, Mayo Clinic, Rochester, Minn., USA). The calyx and renal pelvis were carefully identified and excluded (fig. 1, 2).

The number of cysts in each kidney was counted manually by a radiologist (H.S., with 12 years of experience in body MR imaging). Only circular and spheroid structures $\geq 2 \mathrm{~mm}$ in diameter whose signal intensities were close to that of spinal fluid were considered cysts. Each identified and counted cyst was flagged by an electronic marker using an in-house cyst-labeling program (fig. 1b, $2 b$ ). The total number of cysts flagged with red markers was automatically computed.

\section{Cyst Area Measurements Using a Region-Based Method}

The total area of cysts in each kidney was measured by a different radiologist (J.W.) using a region-based thresholding method $[3,7]$. Cysts that were brighter than the renal parenchyma could be measured by summing pixels with intensity values greater than those of the background renal parenchyma. On each renal MR image slice, a binary signal intensity map was generated by determining a threshold signal intensity that visually distinguished the cyst and renal parenchymal regions. By summing the pixels of white regions in the binary map, the cystic area was measured in each slice.

\section{Semiautomated Cyst Segmentation and Counting}

The semiautomated segmentation program was implemented as an ImageJ plugin [8]. T2-weighted MR images of ADPKD kidneys contain regions of renal cysts (bright in pixel signal intensity) and regions of renal parenchyma (dark in pixel signal intensity). From the signal intensity distribution of the renal cyst and parenchyma pixels, a threshold was automatically determined by an iterative computation using the Iterative Self-Organizing Data Analysis Technique algorithm [9]. A binary image was generated from the threshold that separated renal cyst regions (white) from parenchyma regions (black). If deemed appropriate, the threshold 

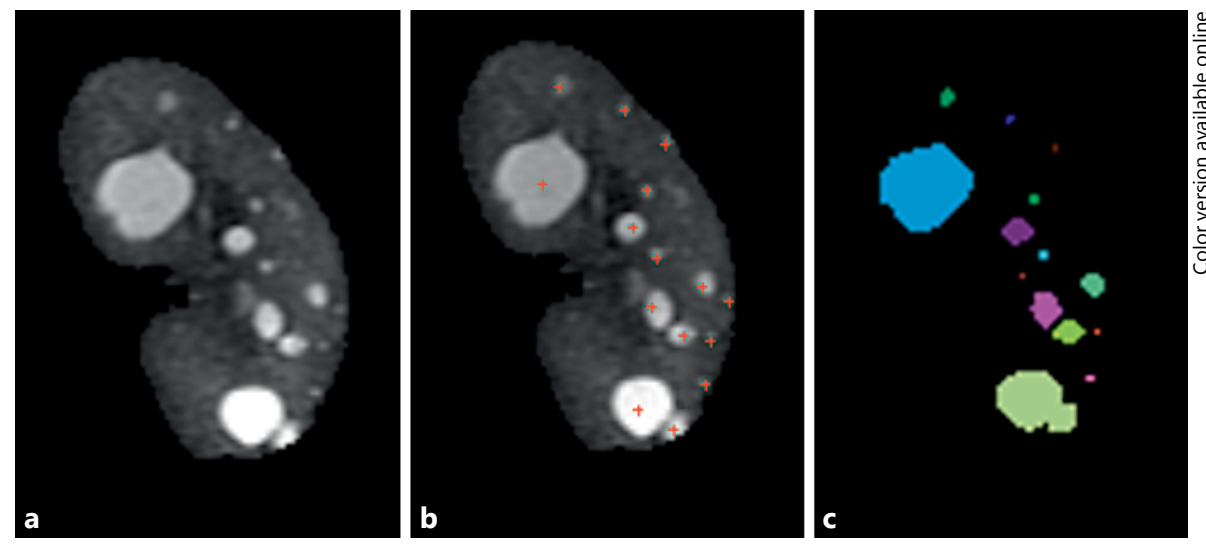

Fig. 1. Mid-slice MR images from an ADPKD patient with mild cyst burden illustrating the initial segmented kidney (a), manual cyst counting (b) and individual cysts segmented using the semiautomated method (c). a Cysts were scattered and discretely defined by surrounding renal parenchyma. Most cysts were well separated without touching each other. $\mathbf{b}$ The manual counting meth- od was performed using an in-house cyst-labeling computer program. Small, poorly defined foci of faint brightness were not counted as cysts because they were difficult to differentiate from background MR image noise in the parenchyma. c Individual renal cysts were segmented and color-coded using the semiautomated method.
Fig. 2. Mid-slice $M R$ images from an ADPKD patient with severe cyst burden illustrating the initial segmented kidney (a), manual cyst counting (b) and individual cysts segmented using the semiautomated method (c). a The kidney was diffusely occupied by numerous cysts with barely discernible renal parenchyma. Some neighboring cysts shared borders. b Individual cysts were manually flagged and counted using an in-house cyst-labeling computer program. c Individual renal cysts were segmented and color-coded using the semiautomated method.
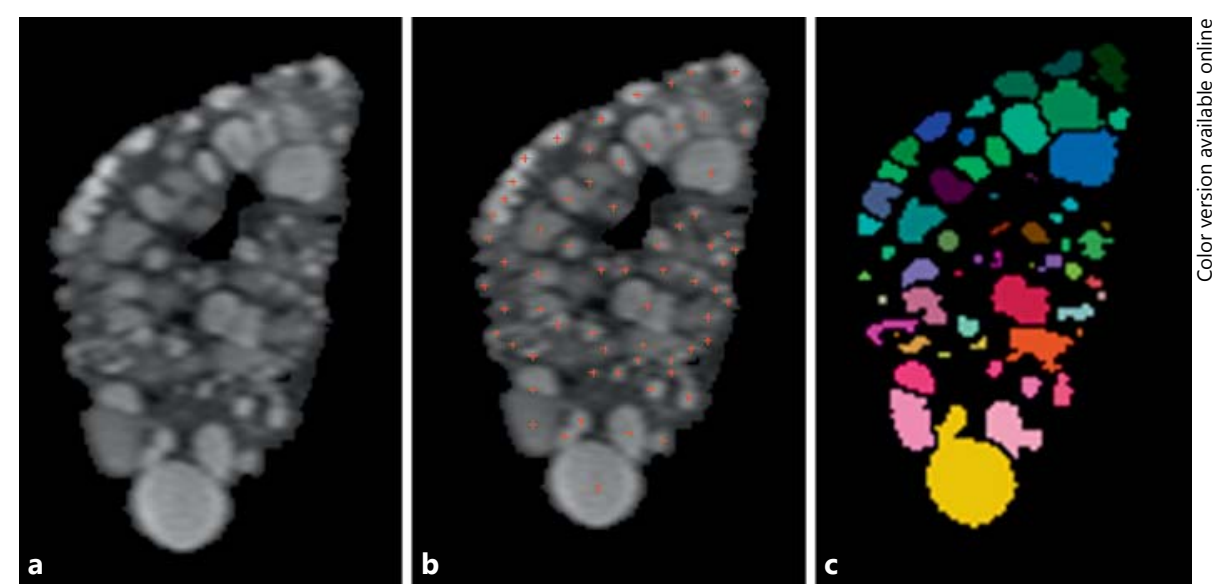

was interactively adjusted by the radiologist (H.S.) to enhance the delineation between renal cyst and parenchyma regions. The renal cyst regions from the binary image were used as the initial candidates and superimposed over the original gray-scale MR image. The cyst candidate regions frequently presented with variable signal intensities due to MR field inhomogeneity and were further revised and filled to retain a relatively uniform signal intensity. A flood fill algorithm was used to replace pixels appearing as holes within the candidate cysts. A connected components analysis was applied to the candidate cyst region to extract and label candidates for individual cyst regions. To further delineate individual cysts from connected components analysis regions, a Euclidean distance map (EDM) was generated from this region. In the EDM, the value of each pixel was calculated to represent the distance to the nearest background pixel. By incorporating watershed segmentation into the EDM, loosely connected cysts were split at their necks [10]. A uniquely identifiable label and color was assigned to each connected component (fig. 1c, 2c). The area and count of individual cysts were automatically computed from the segmented cysts.

\section{Data Analysis}

The performance of the semiautomated segmentation method was evaluated and compared with the manual method in terms of counting the individual cysts in each kidney. The segmentation of each individual cyst could not be assessed because it was not available by the manual method. The level of agreement in cyst counts in each kidney between the semiautomated and manual method was assessed by means of intraclass correlation (ICC) [11] and corresponding confidence intervals (CIs). We also analyzed the interreader reliability in cyst counting between two readers using ICC. The cyst area measurements in each kidney from the semiautomated and region-growing methods were compared by means of a Spearman correlation. In addition, Bland-Altman 
analysis was used to visually assess systemic differences in cyst counting [12] and to estimate the bias and limits of agreement (LOA). The statistical analysis was performed in Stata (version 12, Stata software); Bland-Altman plots were created using the batplot command.

\section{Results}

For all CRISP participants, we successfully segmented individual renal cysts with the semiautomated method. The number of cysts measured with the semiautomated segmentation method ranged from 2 to 69 (mean 26.0) for the right kidney, with a median count of 25 (interquartile range of $16-33$, with the middle $90 \%$ falling between 8 and 52), and 1 to 63 (mean 27.5) for the left kidney, with a median count of 26 (interquartile range of 18-35, with the middle 90\% falling between 8 and 49). The number of cysts determined by the manual counting method ranged from 1 to 69 (mean 25.4) for the right kidney, with a median count of 23 (interquartile range of 15-33, with the middle 90\% falling between 6 and 53), and 1 to 68 (mean 27.6) for the left kidney, with a median count of 27 (interquartile range of 18-36, with the middle $90 \%$ falling between 7 and 52).

The average difference in the total numbers of cysts, i.e. the manual minus the semiautomated segmentation count, was very small, namely -0.52 (SD 3.9, interquartile range -3 to 2 ) for the right kidney and 0.13 (SD 3.9, interquartile range -3 to 3 ) for the left kidney. However, the variability for some individual subjects was not trivial. Six of 241 participants $(2.5 \%)$ had a difference in the total cyst count of greater than 15 , and the middle $90 \%$ of the distribution had differences of 10 or less; as noted by the interquartile range, most differences were 3 or less. The two methods correlated well (right kidney: ICC 0.96, 95\% CI 0.95-0.97; left kidney: ICC 0.96, 95\% CI 0.95-0.97; right and left kidneys combined: ICC 0.97, 95\% CI 0.96-0.98; fig. 3). Although we found a high level of average agreement and overall variability as demonstrated by the high ICCs, Bland-Altman analyses revealed large differences for some individual measurements. More specifically, the LOA were -8.1 to 7.1 for the right side and -7.5 to 7.7 for the left side. Figures $4 \mathrm{a}$ and $\mathrm{b}$ show a relative spread of differences between the LOA over most of the range (e.g. for average counts above 10), with some outliers in the differences (with 1.7 and $3.7 \%$ outside the LOA for the right and left sides, respectively).

The cyst area measurements between the semiautomated segmentation and region-growing method were highly correlated, with a Spearman correlation of 0.958 and a mean difference of -1.025 (semiautomated method minus region-growing method; SD 11.35). For the interreader reliability, the analysis (using log-transformed data) showed that the ICC between the two readers was very high, with an estimate of 0.989 (95\% CI 0.986-0.992). The mean difference (in the original scale) between the readers was nearly 0 for both the left (mean 0.004 , SD 2.50 ) and right sides (mean 0.241, SD 2.74). The middle half of the differences was 2 or less in magnitude (with a total range up to a difference of 9 ).

\section{Discussion}

CRISP demonstrated that both the total renal volume and total cyst volume could be reliably measured by MR imaging and used to monitor the disease progression of ADPKD $[3,4]$. In addition to volumetric measurements, other renal cyst measurements, including the number and size of cysts, might be closely related to ADPKD pathogenesis and the genetic variations found in the family members of patients with $\operatorname{ADPKD}[5,13,14]$. In particular, Harris et al. [5] reported that PKD1 kidneys have a greater number of cysts than PKD2 kidneys and that PKD1 has a more severe disease progression because more cysts develop earlier, not because they grow faster, implicating the disease gene in cyst initiation but not expansion. In an MR imaging study of a pediatric ADPKD cohort, Cadnapaphornchai et al. [15] found that cyst number increases more rapidly in hypertensive ADPKD children. The number of renal cysts was estimated in these two studies by manual counting on a mid-slice of MR images.

A reliable method for measuring cyst numbers in multiple MR image slices and changes over time is important to accurately evaluate the disease progression in ADPKD, particularly when the longitudinal change in the total kidney volume is relatively small, e.g. in children. Although the manual cyst counting method may be straightforward and easy to perform for ADPKD kidneys, it is likely subject to high variability and errors, particularly in kidneys with a severe cyst burden.

The semiautomated and manual methods showed excellent ICCs with relatively small paired differences, although there were some large differences for a small percentage of the measurements. We reviewed these cases to investigate the causes for these large differences. When a number of cysts are weakly discernible from the background renal parenchyma, the segmentation outcome of 
Fig. 3. Scatter plot of manual versus semiautomated renal cyst counts for the right (a) and left kidney (b). The two methods correlated well (right kidney: ICC 0.96, 95\% CI 0.95-0.97; left kidney: ICC 0.96, 95\% CI 0.95-0.97). Diagonal lines represent the line of identity.

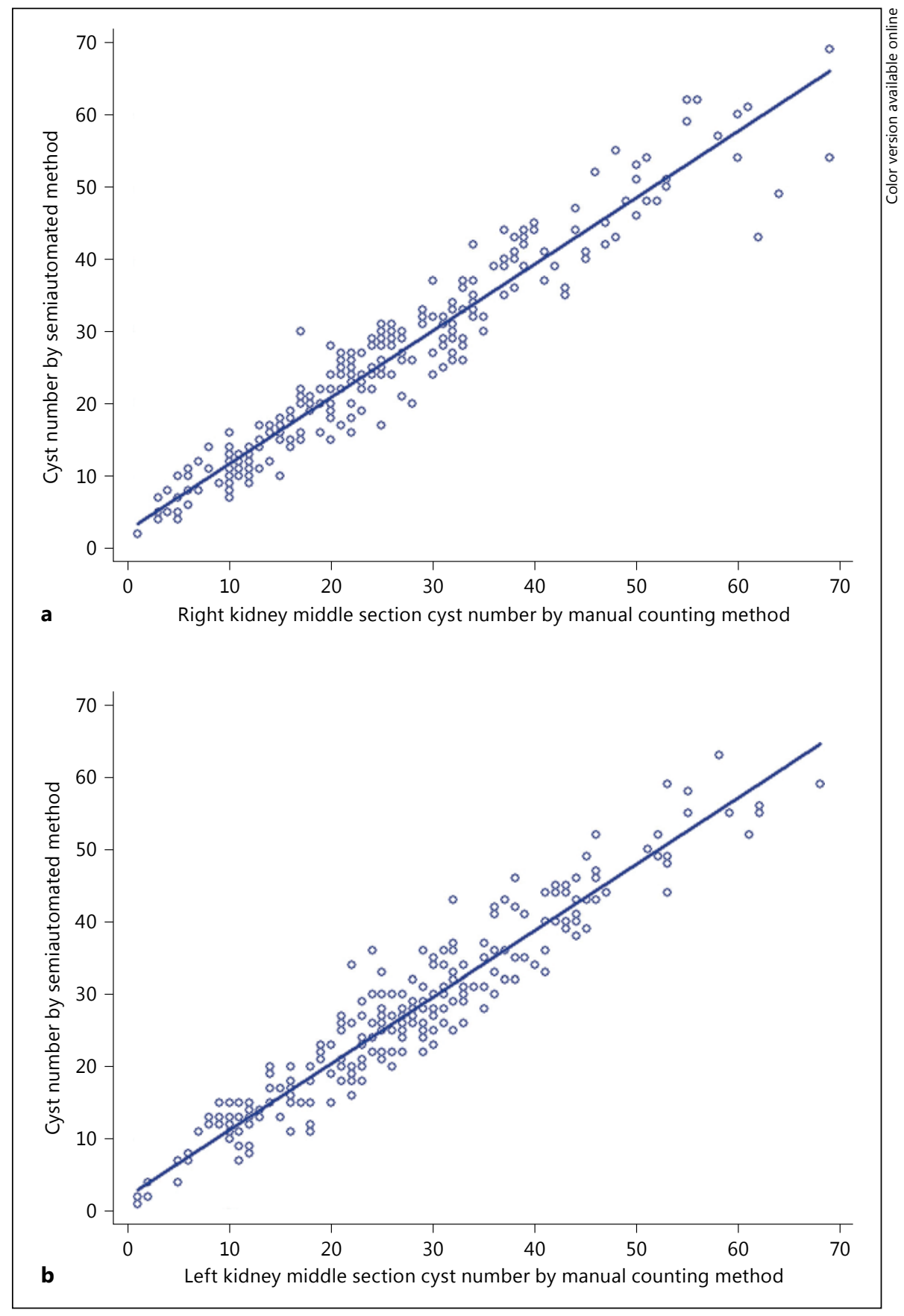

these cysts is highly sensitive to the threshold value, which is adjusted and determined by the radiologist on each image to differentiate cysts from the background parenchyma. There are also regional variations in MR signal intensity within a kidney that may not be uniformly resolved by a single global threshold value. As a result, a considerable number of faintly defined cysts may appear or disappear with a subtle adjustment of a threshold.
On the other hand, manual counting relies on human perception, which is subjective, but at the same time more adaptable to regional signal intensity fluctuations within the kidney. The majority of large differences between the two methods are likely attributable to the intrinsic signal intensity heterogeneity of cysts. One potential approach to identify these cases with large signal intensity heterogeneity would be to compute and analyze 
Fig. 4. Bland-Altman plot for manual versus semiautomated renal cyst counts for the right (a) and left kidney (b).

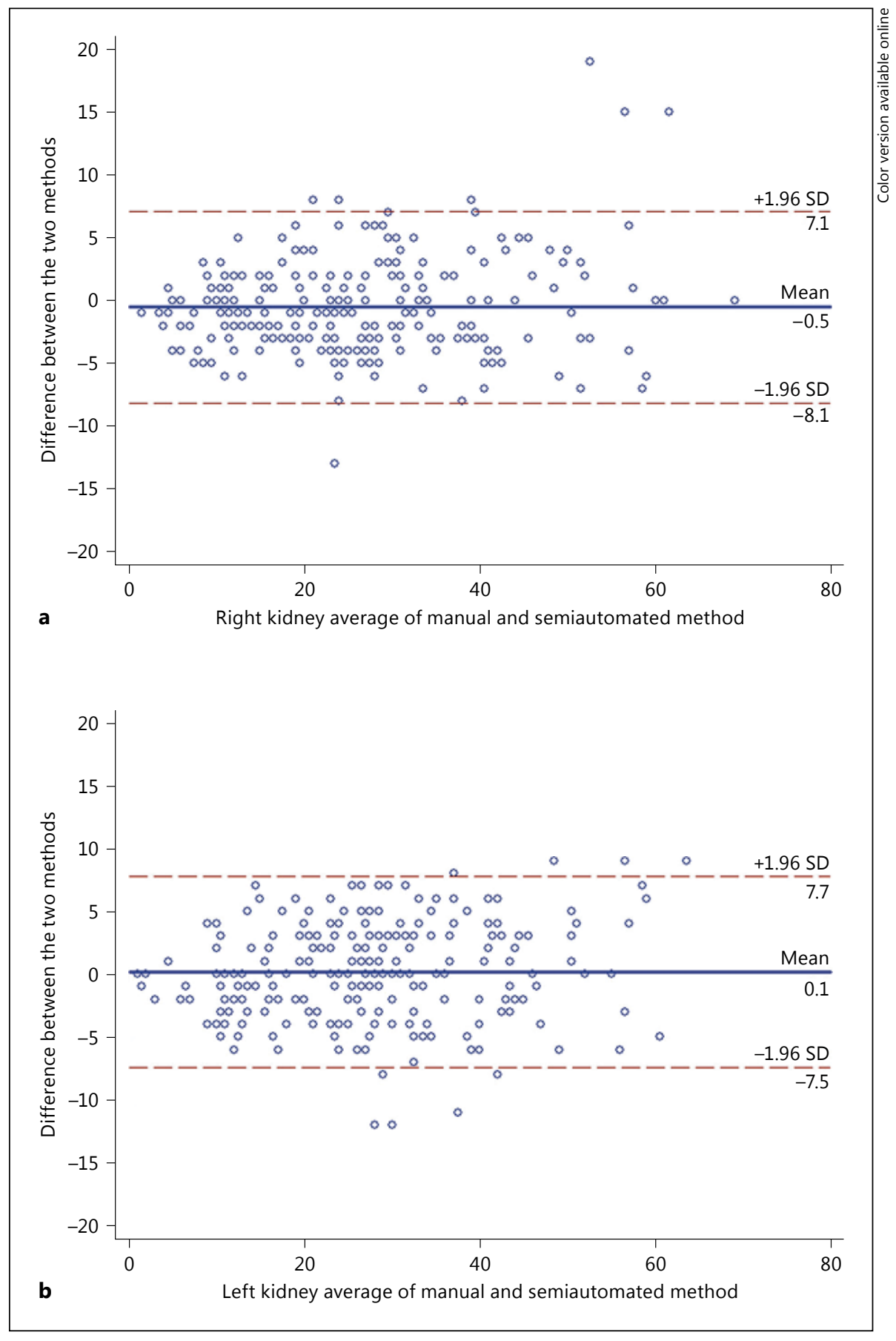

the intensity histogram covering the entire kidney region for the spread of the intensity distribution and to use well-defined simple cysts as the reference to normalize MR signal intensities. Some other differences between the two methods may be related to image quality, which could be improved with further technical advances in the future.
The semiautomated method provides several key advantages. For example, the ability to segment individual cysts by the semiautomated method allows us to study the characteristics of renal cysts in ADPKD. We can investigate both the size and spatial (e.g. medullary vs. cortical) distribution of cysts from the segmented individual cysts within the kidney. While unnecessary for small 
kidneys with a few cysts that can be counted easily, for large kidneys with numerous cysts, the semiautomated method is faster and more efficient than the manual method. In the CRISP cohort, most of the kidneys contained numerous renal cysts, and thus the semiautomated method was preferred by the radiologists. Since no specific reference measurement was available to compare each individual renal cyst, the size and locational accuracy of segmentation of individual cysts could not be assessed.

Although counting cysts from a 3D MR image set representing the whole kidney volume is preferred to counting only from limited MR image slices, manually counting numerous cysts on a 3D MR image set is exceedingly laborious and may not be accurate [16]. A recent semiautomated method for cyst segmentation and counting from a 3D kidney volume MR image set was reported to have a relatively good performance for kidneys with mild and moderate cystic burdens [6]. However, the 3D semiautomated method failed to reliably segment individual cysts in advanced ADPKD kidneys, in which the renal parenchyma is largely replaced by innumerable cysts. These cysts are touching and inseparable from each other, without discernible background renal parenchyma. The segmentation of cysts in advanced ADPKD, which requires a labor-intensive manual editing of each cyst even after an initial segmentation by computer, is impractical. On the other hand, our 2D segmentation method based on mid-slice MR images was successful for the entire spectrum of cyst severity in all CRISP participants. Although this method is limited to an area as opposed to a volumetric data set, it could be applied to additional MR slices sampled over a volumetric data set (e.g. two additional slices corresponding to the mid-slice of the anterior half and the mid-slice of the posterior half of the series) to approximate and estimate the number of individual cysts in a 3D MR imaging representation of the kidney. We investigated this issue further for a small subset of the participants (16 cases) by comparing the $3 \mathrm{D}$ individual cyst count to the estimated cyst count from the mid-slice times the number of slices in each kidney; results yielded a correlation of 0.80 , and a linear regression (of log-transformed counts, with the product of the midslice count times the number of slices as the predictor) gave similar predictions. The mean predicted count was 70.0 (SD 19.9), versus the actual mean count of 71.9 (SD 21.7 ), to give a resulting mean difference of -1.83 (SD 16.2).

The semiautomated method we developed requires a prior segmentation of the kidney from abdominal MR images. There are a number of image segmentation methods for the kidney published in the literature, ranging from simple manual delineation to the application of advanced object detection algorithms [17]. In our approach, we focused on the development of a simple and robust segmentation scheme to minimize the operator dependence. The kidney was segmented semiautomatically in each slice of the MR images by placing a seed point over the kidney area. Renal hila and the extraparenchymal collecting system were edited and excluded by an operator. The segmented kidney was also used to estimate the kidney volume. Although a fully automated segmentation method would be preferred for the segmentation of both the kidney and individual renal cysts, this works well only for normal kidneys $[18,19]$. With the progression of ADPKD, the morphology and MR signal intensities of kidneys become too complex to reliably segment the kidney and individual cysts without monitoring and editing by an expert radiologist [20].

This study has some limitations. Firstly, the semiautomated method focused on 2D images, which do not include the total number of cysts in the whole kidney. Secondly, in this analysis, we identified and subdivided cyst brightness in T2-weighted MR images. Complex and hemorrhagic cysts may appear as a gray or dark signal on T2-weighted images and cannot be identified or segmented in any study using either manual or semiautomated methods. These cysts need to be carefully identified in the visualization and comparison of $\mathrm{T} 1$ - and $\mathrm{T} 2$-weighted images. Thirdly, microscopic cysts are 'invisible' by MR imaging and cannot be segmented. A recent study showed that a large number of microscopic cysts in the ADPKD kidney cannot be detected in vivo with MR imaging because of imaging resolution limitations [16]. Nevertheless, the collective volume of these tiny cysts in relation to the overall kidney size is relatively insignificant [16]. Finally, even if the segmentation process is semiautomated, it may still require a manual edit of the threshold after the initial segmentation by computer. When renal cysts are overestimated or not labeled automatically by the program, the radiologist edits the threshold on MR images manually in order to eliminate an incorrectly segmented structure or to add new cysts.

In conclusion, we have developed a semiautomated method to segment and count renal cysts from a mid-slice of an MR image data set in ADPKD kidneys with a wide range of cyst severity. This method facilitates the assessment of the number and spatial distribution of renal cysts for the characterization and evaluation of disease progression of ADPKD. 


\section{Acknowledgements}

The CRISP study is supported by cooperative agreements from the National Institute of Diabetes and Digestive and Kidney Diseases of the National Institutes of Health (DK056943, DK056956, DK056957, DK056961) and by the National Center for Research Resources General Clinical Research Centers at each institution (RR000039, Emory University; RR00585, Mayo College of Medicine; RR23940, Kansas University Medical Center; RR000032, University of Alabama at Birmingham) and the National Center for Research Resources Clinical and Translational Science Awards at each institution (RR025008, Emory University; RR024150, Mayo College of Medicine; RR033179, Kansas University MedicalCenter; RR025777 and UL1TR000165,
University of Alabama at Birmingham; RR024153 and UL1TR000005, University of Pittsburgh School of Medicine). The investigators are indebted to the radiologists, radiology technologists, imaging engineering and study coordinators in CRISP.

\section{Disclosure Statement}

K.T.B., A.B.C. and J.J.G. are consultants to Otsuka Corporation, and V.E.T. received research support from Otsuka Corporation. M.M. is a consultant to Otsuka Corporation and Alexion Pharmaceuticals.

\section{References}

1 Chapman AB, Bost JE, Torres VE, GuayWoodford L, Bae KT, Landsittel D, Li J, King BF, Martin D, Wetzel LH, Lockhart ME, Harris PC, Moxey-Mims M, Flessner M, Bennett WM, Grantham JJ: Kidney volume and functional outcomes in autosomal dominant polycystic kidney disease. Clin J Am Soc Nephrol 2012;7:479-486.

2 Wilson PD: Polycystic kidney disease. N Engl J Med 2004;350:151-164.

-3 Chapman AB, Guay-Woodford LM, Grantham JJ, Torres VE, Bae KT, Baumgarten DA, Kenney PJ, King BF Jr, Glockner JF, Wetzel LH, Brummer ME, O’Neill WC, Robbin ML, Bennett WM, Klahr S, Hirschman GH, Kimmel PL, Thompson PA, Miller JP: Renal structure in early autosomal-dominant polycystic kidney disease (ADPKD): The Consortium for Radiologic Imaging Studies of Polycystic Kidney Disease (CRISP) cohort. Kidney Int 2003; 64:1035-1045.

4 Grantham JJ, Torres VE, Chapman AB, Guay-Woodford LM, Bae KT, King BF Jr, Wetzel LH, Baumgarten DA, Kenney PJ, Harris PC, Klahr S, Bennett WM, Hirschman GN, Meyers CM, Zhang X, Zhu F, Miller JP: Volume progression in polycystic kidney disease. N Engl J Med 2006;354:2122-2130.

$\checkmark 5$ Harris PC, Bae KT, Rossetti S, Torres VE, Grantham JJ, Chapman AB, Guay-Woodford LM, King BF, Wetzel LH, Baumgarten DA, Kenney PJ, Consugar M, Klahr S, Bennett WM, Meyers CM, Zhang QJ, Thompson PA, Zhu F, Miller JP: Cyst number but not the rate of cystic growth is associated with the mutated gene in autosomal dominant polycystic kidney disease. J Am Soc Nephrol 2006;17: 3013-3019.
6 Bae K, Park B, Sun H, Wang J, Tao C, Chapman AB, Torres VE, Grantham JJ, Mrug M, Bennett WM, Flessner MF, Landsittel DP, Bae KT: Segmentation of individual renal cysts from MR images in patients with autosomal dominant polycystic kidney disease. Clin J Am Soc Nephrol 2013;8:1089-1097.

7 Bae KT, Zhu F, Chapman AB, Torres VE, Grantham JJ, Guay-Woodford LM, Baumgarten DA, King BF Jr, Wetzel LH, Kenney PJ, Brummer ME, Bennett WM, Klahr S, Meyers CM, Zhang X, Thompson PA, Miller JP: Magnetic resonance imaging evaluation of hepatic cysts in early autosomal-dominant polycystic kidney disease: the Consortium for Radiologic Imaging Studies of Polycystic Kidney Disease cohort. Clin J Am Soc Nephrol 2006;1: 64-69.

8 Abramoff MD, Magalhaes PJ, Ram SJ: Image processing with ImageJ. Biophotonics International 2004;11:36-42.

$>9$ Ridler TW, Calvard S: Picture thresholding using an iterative selection method. IEEE Trans Syst Man Cybern 1978;8:630-632.

10 Leymarie F, Levine MD: Fast raster scan distance propagation on the discrete rectangular lattice. CVGIP Image Understanding 1992; 55:84-94.

11 Shrout PE, Fleiss JL: Intraclass correlations: uses in assessing rater reliability. Psychol Bull 1979;86:420-428.

12 Bland JM, Altman DG: Statistical methods for assessing agreement between two methods of clinical measurement. Lancet 1986;i:307-310.

13 Fick-Brosnahan GM, Belz MM, McFann KK, Johnson AM, Schrier RW: Relationship between renal volume growth and renal function in autosomal dominant polycystic kidney disease: a longitudinal study. Am J Kidney Dis 2002;39:1127-1134.
14 Grantham JJ, Cook LT, Torres VE, Bost JE, Chapman AB, Harris PC, Guay-Woodford LM, Bae KT: Determinants of renal volume in autosomal-dominant polycystic kidney disease. Kidney Int 2008;73:108-116.

15 Cadnapaphornchai MA, Masoumi A, Strain JD, McFann K, Schrier RW: Magnetic resonance imaging of kidney and cyst volume in children with ADPKD. Clin J Am Soc Nephrol 2011;6:369-376.

16 Grantham JJ, Mulamalla S, Grantham CJ, Wallace DP, Cook LT, Wetzel LH, Fields TA, Bae KT: Detected renal cysts are tips of the iceberg in adults with ADPKD. Clin J Am Soc Nephrol 2012;7:1087-1093.

17 Zollner FG, Svarstad E, Munthe-Kaas AZ, Schad LR, Lundervold A, Rorvik J: Assessment of kidney volumes from MRI: acquisition and segmentation techniques. AJR Am J Roentgenol 2012;199:1060-1069.

$\checkmark 18$ Shim H, Chang S, Tao C, Wang JH, Kaya D, Bae KT: Semiautomated segmentation of kidney from high-resolution multidetector computed tomography images using a graph-cuts technique. J Comput Assist Tomogr 2009;33: 893-901.

19 Khalifa F, Elnakib A, Beache GM, Gimel'farb G, El-Ghar MA, Ouseph R, Sokhadze G, Manning S, McClure P, El-Baz A: 3D kidney segmentation from CT images using a level set approach guided by a novel stochastic speed function. Med Image Comput Comput Assist Interv 2011;14:587-594.

20 Bae KT, Commean PK, Lee J: Volumetric measurement of renal cysts and parenchyma using MRI: phantoms and patients with polycystic kidney disease. J Comput Assist Tomogr 2000;24:614-619. 